\title{
O ESTADO DA ARTE DA ABORDAGEM DIREITO E POLÍTICAS PÚBLICAS EM ÂMBITO INTERNACIONAL: PRIMEIRAS APROXIMAÇÕES
}

\section{THE STATE OF THE ART OF THE LAW AND PUBLIC POLICY (LPP) APPROACH IN INTERNATIONAL BIBLIOGRAPHY}

\author{
MATHEUS SILVEIRA DE SOUZA ${ }^{1}$ \\ MARIA PAULA DALLARI BUCCI ${ }^{2}$
}

RESUMO: O artigo relata a produção internacional sobre direito e políticas públicas, a partir de levantamento bibliográfico focado em produção que destacasse questões metodológicas e as especificidades disciplinares para subsidiar a abordagem Direito e Políticas Públicas (DPP). Apresenta-se a produção acadêmica dos principais autores identificados, seja em razão da densidade do debate específico, seja em função de conexões pertinentes entre questões analisadas pelos autores e as perguntas que vem norteando a construção da abordagem DPP no Brasil. Justificados os critérios de seleção, faz-se um breve relato de trabalhos de William Clune, Kreis \& Christensen, Kiyoung Kim, Barclay \& Birkland e Lasswell \& McDougal. Desses, o material de maior relevância, em função de sua densidade, concentra-se nos artigos de Clune. Resenha-se a discussão do autor sobre a instrumentalização do direito pela política com vistas à efetivação de políticas públicas no Welfare State e se analisam técnicas de pesquisa e quadros de análise úteis à abordagem DPP (CLUNE, 1993). Posteriormente, discute-se, a partir de Kreis \& Christensen, a correlação existente entre as diferentes espécies normativas na formação e implementação das políticas públicas, tendo em vista que, a depender da arena de que a norma tenha emanado - Executivo, Legislativo ou Judiciário - haverá efeitos relevantes para a efetividade da política, considerando diferentes pontos de veto e distintos desenhos jurídico-institucionais. Na parte final, noticiam-se debates interdisciplinares na fronteira dessa abordagem, explicitando-se as discussões travadas entre estudiosos de políticas públicas e

\footnotetext{
${ }^{1}$ Mestrando em Direito do Estado pela Faculdade de Direito da Universidade de São Paulo - FDUSP (Brasil). E-mail: mathmss94@gmail.com. Currículo Lattes: http://lattes.cnpq.br/1967841099775744. ORCID n. 0000-0002-6754-9386.

${ }^{2}$ Professora Associada da Faculdade de Direito da Universidade de São Paulo - FDUSP (Brasil). Email: mariapaula@usp.br. Currículo Lattes: http://lattes.cnpq.br/5277262878389044. ORCID n. 0000-0003-2862-8986.
} 
pesquisadores da área jurídica sobre os policymakers (BARCLAY; BIRKLAND, 1998).

Palavras-Chave: Direito e Políticas Públicas; Estado da arte; Estado social e Políticas Públicas.

ABSTRACT: The article reports the international production on law and public policies, based on a bibliographic survey focused on production that highlighted methodological issues and disciplinary specificities to support the Law and Public Policy (LPP) approach. The academic production of the main authors identified is presented, either because of the density of the specific debate, or because of pertinent connections between issues analyzed by the authors and the questions that have been guiding the construction of the LPP approach in Brazil. Justifying the selection criteria, a brief account of works by William Clune, Kreis \& Christensen, Kiyoung Kim, Barclay \& Birkland, and Lasswell \& McDougal is given. Of these, the most relevant material, due to its density, is concentrated in Clune's articles. The author's discussion on the instrumentalization of law by politics with a view to the implementation of public policies in the Welfare State is reviewed and research techniques and analysis frameworks useful to the DPP approach are analyzed (CLUNE, 1993). Subsequently, from Kreis \& Christensen, the correlation between the different normative species in the formation and implementation of public policies is discussed, considering that, depending on the arena from which the standard originated - Executive, Legislative or Judicial - there will be relevant effects for the effectiveness of the policy, considering different points of veto and different legal-institutional designs. In the final part, interdisciplinary debates are reported at the frontier of this approach, making explicit the discussions between policymakers and legal researchers about policymakers (BARCLAY; BIRKLAND, 1998).

KeYWORDS: Law and Public Policy; State of the art; Welfare state and public policy.

\section{INTRODUÇÃO}

A política pública é um objeto de estudo intrinsecamente interdisciplinar ${ }^{3}$, de tal modo que é possível enxergá-la por lentes teóricas advindas das mais variadas

\footnotetext{
${ }^{3}$ Ver, a esse respeito, as noções de multidisciplinaridade e interdisciplinaridade de Eduardo Marques e Carlos Aurélio Pimenta de Faria, referidas no artigo Método e Aplicações da Abordagem Direito e Políticas Públicas, de Maria Paula Dallari Bucci, neste dossiê.
} 
áreas do conhecimento, tais como economia, educação, ciência política, serviço social e outras. Embora o uso de cada lente permita identificar especificidades que ampliam a visualização desse objeto, algumas áreas do conhecimento possuem muitos pontos de conexão entre si, o que dá margem ao surgimento de novas aproximações, como é o caso da abordagem Direito e Políticas Públicas (DPP). Há diversos pontos de conexão entre essas duas áreas, que possibilitam uma progressiva demarcação do objeto da abordagem, tais como o compartilhamento de instituições, as relações entre as políticas públicas e diferentes espécies normativas, a discricionariedade, os processos e seu regramento, a formatação dos desenhos jurídico institucionais, e ainda a instrumentalização do direito para a implementação de políticas públicas.

$\mathrm{Na}$ busca de um método para a evolução dessa abordagem ${ }^{4}$, percebeu-se a importância de um levantamento da produção bibliográfica sobre o assunto fora do Brasil, em países em que a reflexão específica pudesse estar mais desenvolvida. Este artigo é o relato dessa pesquisa e seus principais achados, com um breve balanço sobre a importância dessa produção. ${ }^{5}$

Na construção de um "estado da arte em Direito e Políticas Públicas" em âmbito internacional, percebem-se muitos pontos de contato entres as teorias produzidas em âmbito nacional e internacional. $\mathrm{O}$ imbricamento entre direito, políticas públicas e instituições, a instrumentalização do direito para a efetivação das políticas públicas, a intersecção entre direito e política e a elaboração de arranjos institucionais são exemplos de temáticas encontradas em ambos os contextos.

Em âmbito internacional, o surgimento da abordagem law and public policy pode ser evidenciado pelo número de periódicos que possuem essa temática como ponto central de pesquisa. É possível citar algumas revistas dos EUA, criadas a partir da década de 1980, que buscam expandir a produção bibliográfica nessa área, tais como a Cornell Journal of Law and Public Policy, Yale Law and Policy Review, Stanford

\footnotetext{
${ }^{4}$ Ver o artigo Método e Aplicações da Abordagem Direito e Políticas Públicas, de Maria Paula Dallari Bucci, neste dossiê.

${ }^{5}$ No trabalho de pesquisa, a busca e análise preliminar do material foi realizada por Matheus Silveira de Souza, cabendo a M.Paula Dallari Bucci o desenho do projeto e a orientação dos critérios para coleta e seleção do material. A pesquisa foi financiada com recursos dos direitos autorais do livro Judicialização da Saúde: A visão do Poder Executivo (BUCCI; DUARTE, 2017), mediante cessão expressamente prevista no contrato de edição, pela qual os autores agradecem a todos co-autores daquela obra: Clarice Seixas Duarte, Fernando Aith, Márcia Walquiria Batista dos Santos, Aloysio Vilarino dos Santos, Cristiana Melhado Araújo Lima, Alessandra Pinto Magalhães de Abreu, Ana Carolina Varandas Martos, André Serafim Bernardi, Arthur Souza Rodrigues, Camila Sobrinho Paisano, Luís Gustavo Gomes Primos, Marcos Felipe de Albuquerque Oliveira, Renata Lima Gonçalves, Yeun Soo Cheon, Alessandra Gotti, Patrícia Ribeiro Brito, Patrícia Ulson Pizarro Werner, Luiz Rascovski, Ighor Rafael de Jorge, Raquel Requena Rachid, Paulo Vitor Bergamo Braga, Junia Coelho Lemos, Fernanda Massad de Aguiar Fabretti, Artur Pericles Lima Monteiro e Otávio Augusto Venturini de Sousa.
} 
Law and Policy Review, Harvard Journal of Law and Public Policy, The Georgetown Journal of Law \& Public Policy, entre outros periódicos. ${ }^{6}$

Embora haja diversas revistas que carregam o título Law and Public Policy, é mais frequente encontrarmos nesses periódicos análises aplicadas de políticas públicas, como discussões sobre políticas de saúde ou educação, do que debates sobre questões teóricas ou especificidades metodológicas da abordagem DPP. Esse problema pode ser mais bem compreendido ao observarmos que não há um objeto claro ou fronteiras disciplinares definidas para a atividade de pesquisa sobre ele ${ }^{7}$. A descrição do objetivo dessas revistas, encontradas em seus próprios sites, não parece evidenciar um consenso sobre o que seja a abordagem DPP em termos disciplinares e metodológicos. A Stanford Law and Policy Review tem como objetivo a "publicação de artigos que analisam a intersecção do nosso sistema jurídico com políticas públicas de nível federal, estadual e local" 8 (KREIS; CHRISTENSEN, 2013). A Yale Law and Policy Review tem entre seus objetivos investigar a "intersecção entre direito e políticas públicas". Já o Cornell Journal of Law and Public Policy busca publicar não apenas análises da lei mas também evidenciar o impacto de seu desenvolvimento (KREIS; CHRISTENSEN, 2013).

A partir da década de 1980 foram criados diversos cursos em tradicionais faculdades de direito, denominados como Law and Public Policy Program, que buscavam construir uma abordagem interdisciplinar, vinculando conhecimentos jurídicos com habilidades necessárias à formulação e implementação de políticas públicas. Um exemplo é o Law and Public Policy (LPP) criado na Stanford Law School, que abarca questões ligadas aos direitos humanos, agências de proteção ambiental, políticas de saúde, desenho de conselhos, políticas criminais etc, explorando as relações existentes entre políticas públicas e direito. Destaca-se, ainda, o The Law and Public Policy Program, criado no âmbito da Beasley School of Law, bem como o Law and Public Policy Program concebido na Catholic University of America. O último possibilita aos alunos analisar as interações entre as atividades legislativas, judiciais e regulatórias que ocorrem em Washington DC e envolvem direito e políticas públicas (McDOUGAL, 1988). Como objetivo geral, esses cursos pretendem conferir aos estudantes de direito o desenvolvimento de habilidades necessárias para a atuação em carreiras vinculadas às políticas públicas, como advocacy, formulação de políticas, entre outras.

A partir desse pano de fundo, o artigo objetiva realizar uma primeira aproximação sobre o estado da arte de DPP em âmbito internacional, apresentando

${ }^{6}$ Os periódicos citados foram escolhidos em virtude da sua relevância acadêmica, além das referências em KREIS e CHRISTENSEN.

${ }^{7}$ Sobre a discussão de direito e políticas públicas como campo ou abordagem, ver artigo de M. Paula Dallari Bucci neste dossiê.

${ }^{8}$ As traduções do original em inglês são de responsabilidade dos autores que assinam o presente artigo. Os termos de uso consolidado em inglês foram mantidos nessa língua. 
os trabalhos dos principais autores identificados, selecionados por abordarem direito e políticas públicas nas suas especificidades disciplinares e metodológicas, e não apenas para a análise de políticas públicas setoriais. São eles: William Clune, Kreis \& Christensen, Kiyoung Kim, Barclay \& Birkland, Hensler, Sarat \& Silbey e Lasswell \& McDougal. ${ }^{9}$

Considerando o contexto geopolítico em que o Brasil está inserido, a pesquisa abrangeu também a produção sobre DPP na bibliografia latinoamericana, encontrando contribuições relevantes em Víctor Abramovich (2006; 2009), Laura Pautassi (2009a; 2009b) e Gorki G. Mantilla (2010), entre outros. Entretanto, pela limitação de espaço, essa bibliografia não será examinada aqui.

\section{A CONTRibuição de William Clune PaRA A ABordagem Direito E Políticas Públicas}

William Clune é Professor Emérito da University of Wisconsin Law School e desenvolve pesquisas sobre políticas públicas de educação, financiamento escolar, implementação, entre outros temas. Na década de 80 , o autor começou a se dedicar à temática direito e políticas públicas, tendo escrito ao menos três artigos relevantes sobre o assunto ${ }^{10}$. O primeiro, intitulado A Political Model of Implementation and Implications of the Model for Public Policy, Research, and the Changing Roles of Law and Lawyers, foi publicado em 1983 e apresenta um modelo de implementação envolvendo atores governamentais e não governamentais, instituições, ideologia, indução de comportamento etc. - que destaca a importância do direito nessa fase da política pública e evidencia sua imbricação com o campo político (CLUNE, 1983).

Posteriormente, em 1993, Clune escreve Law and Public Policy: map of an area, traçando um panorama do tema direito e políticas públicas, em que discorre sobre as diferenças e semelhanças das duas áreas do conhecimento, as teorias e lógicas subjacentes a cada uma, e ainda, apresentando diferentes quadros de análise utilizados nessa abordagem (1993).

No último artigo sobre o tema, intitulado Legal Disintegration and $a$ Theory of the State, de $2011^{11}$, o professor de Wisconsin aborda as mudanças ocorridas no âmbito da Teoria do Estado, diferenciando o pensamento jurídico dominante daquele

\footnotetext{
9 A pesquisa examinou as seguintes bases de dados: Jstor, HeinOnline, Web of Science e Scielo Periódicos. Dentro dos critérios de seleção, foram identificados predominantemente autores norteamericanos. Os artigos encontrados datam, em sua maioria, da década de 1980 e 1990, mas há algumas referências importantes de datas anteriores, como Lasswell e McDougal (1943), e posteriores, como Lowi (2003), Kreis e Christensen (2013) e Kiyoung Kim (2014).

${ }_{10}$ Alguns dos trabalhos de Clune foram analisados de maneira mais aprofundada nos artigos de Maria Paula Dallari Bucci e Emiliano Brunet, neste dossiê.

${ }^{11}$ Na verdade, o artigo fora publicado inicialmente em 1989, conforme nota 105 em Law and Public Policy: map of an area.
} 
chamado de "periférico", no qual se inseriria a policy analysis, como expediente do pensamento progressista. Ao final, o autor entende que a teoria central e a periférica se complementariam, segundo o que descreve como a reintegração da teoria estatal (CLUNE, 2011). Em seguida, apresenta-se breve resenha desses três artigos selecionados William Clune.

\section{DifERENÇAS E APROXIMAÇÕES ENTRE DUAS ÁREAS DO CONHECIMENTO}

De acordo com Clune, pode haver certa redundância nos termos direito e políticas públicas, considerando que o direito também é uma política pública, enquanto vontade coletiva da sociedade expressa em normas vinculantes. De modo similar, toda política pública é direito, pois aquela dependerá desse ou das instituições jurídicas em algum momento da sua existência (CLUNE, 1993). A norma responsável por formalizar a existência jurídica de uma política pública, ou ainda, o desenho jurídico institucional de uma policy criada pelo direito são exemplos dessa imbricação.

Ainda que haja um íntimo vínculo entre as duas áreas, algumas características podem auxiliar na demarcação de diferenças entre ambas. Segundo o autor, enquanto o direito tradicionalmente busca proteger interesses particulares, olhando para a justiça individual, a política pública preocupa-se com o bem estar de um grupo, de forma agregada, considerando que essa nunca é construída para uma única pessoa, mas no mínimo para um grupo de pessoas. Embora algumas áreas do direito estejam vinculadas à noção de bem estar coletivo, como o direito ambiental e o direito constitucional, o próprio exercício da advocacia se preocupa, tradicionalmente (mas não apenas), com o bem estar individual de um cliente. Assim, haveria uma aparente dicotomia entre bem estar em escala e bem estar individual. Entretanto, Clune indica que a realização e a proteção de direitos individuais necessitam, não raramente, de uma infraestrutura prévia de políticas públicas, o que evidencia uma diferenciação que ao invés de distanciar, aproxima as duas áreas (CLUNE, 1993).

\subsection{Formalismo jurídico e policy analysis}

As políticas públicas são orientadas pela busca de resultados, almejando produzir efeitos concretos na realidade, não sendo estabelecidas, necessariamente, a partir de concepções de justiça de âmbito filosófico. Essa diferenciação se dá, principalmente, pelas diferenças existentes entre o formalismo jurídico e o consequencialismo (CLUNE, 1993).

Ao analisar os distintos tipos de justificação e racionalização subjacentes ao direito, Clune afirma que "o raciocínio jurídico é conceitual, ético e analógico; e repousa (paradoxalmente) em uma visão de mundo ética, uma imagem de uma sociedade justa e ordenada" (CLUNE, 2011). Por outro lado, o raciocínio vinculado à policy analysis é "consequencialista (apoiando-se em previsões sobre 
comportamento social, incluindo tudo, desde desvios sociais até orçamentos equilibrados)" (CLUNE, 2011, p. 190), e constitui uma forma de instrumentalização do direito direcionada a solucionar problemas sociais. Uma breve análise dos distintos grupos vinculados à advocacy que atuam no Poder Legislativo para a transformação de seus interesses em normas parece fragilizar a concepção do direito enquanto fonte pura de valores sociais. A aproximação entre direito e política, assim, permite uma ampliação da análise jurídica.

A policy analysis também requer um conhecimento específico de determinadas áreas da sociedade - saúde, educação, segurança pública - para a elaboração das políticas. Dessa forma, a análise de políticas públicas aplicada no campo jurídico tem uma preocupação mais focada nos resultados produzidos pela ação governamental, considerando os objetivos previamente determinados e os problemas que se pretende solucionar com as políticas públicas. Essa noção da policy analysis vinculada ao consequencialismo carrega a premissa da instrumentalização do direito. De acordo com o professor de Wisconsin, esse caráter instrumental diminuiria a característica do direito - encontrado na modernidade - enquanto fonte de valores da sociedade. Se o direito é instrumentalizado para resolver problemas, ele acaba perdendo sua estabilidade enquanto representação dos valores sociais (CLUNE, 1983).

Avançando nessa discussão, o autor defende que uma possibilidade de reintegração entre as duas teorias poderia ser encontrada em uma nova forma de policy analysis, que dê mais atenção à participação e ao empoderamento do que modelos de implementação top/down ou comando e controle (command control). Nesse ponto, requer-se uma posição ativa dos grupos sociais, através da distribuição de recursos políticos, financeiros e culturais (CLUNE, 2011).

\subsection{Direção centralizada e ação autônoma}

Um paradoxo relevante que caracteriza as políticas públicas é que ao mesmo tempo em que essas são dirigidas de forma centralizada, elas também necessitam de uma ação descentralizada para alcançar os objetivos que propõem. Assim, Clune se apoia no trabalho de Teubner (1988) para afirmar que ambas as características carregam uma tensão entre si, de modo que: "essa tensão entre a direção centralizada e a ação autônoma é o problema central da política pública". (1993, p. 4). Essa contradição vincula-se à necessidade do Estado de resolver os problemas sociais ao mesmo tempo em que respeita a autonomia individual dos cidadãos. Clune (1993, p. 12) coloca a questão dessa forma: "como guiar atores autônomos sem destruir sua autonomia?".

Esse paradoxo também envolve a possibilidade de o Estado estimular a prática de condutas por parte dos indivíduos, a partir da previsão de políticas 
preventivas ${ }^{12}$. Incentivos estatais para as empresas que diminuam a poluição ambiental, por exemplo, envolvem uma determinação central que depende de uma ação autônoma por parte daquelas. Esse modelo de interação entre controle central e ação descentralizada é referido como "bargaining in the shadow of the law". A estratégia, segundo Clune (1993), seria utilizar o poder de barganha do direito para direcionar as pessoas no sentido de um bem estar coletivo.

\section{O ESTADo SOCIAL E A PERMANÊNCIA DAS INSTITUIÇÕES}

\subsection{O Direito e as Políticas Públicas no contexto do Estado Social}

A política pública, tal qual descrita pelo autor, não tem uma existência atemporal e deslocada de um contexto histórico e social, mas pelo contrário, é fruto de uma forma específica de organização do Estado e da sociedade. Assim, a política pública, como a conhecemos atualmente, tem como fonte o Welfare State e a organização moderna da economia capitalista. A própria alteração na postura do Estado social, que deve atuar de forma prestacional para concretizar direitos sociais, requer um aprofundamento em relação às políticas públicas. A ação governamental em escala ampla - indispensável para a elaboração e implementação das políticas públicas - seria fruto das enormes infraestruturas requeridas pelos sistemas de produção modernos, os quais, por sua vez, são amplos e dinâmicos (CLUNE, 1993).

Clune destaca a relação de interdependência entre o direito, as políticas públicas

e a economia, afirmando que as policies se preocupam, intensamente, com a produção, o consumo e altos níveis de desempenho econômico. Problemas relativos ao desemprego, desenvolvimento sustentável, propriedade, assistência social, regulação da atividade empresarial e redistribuição de renda suscitam a atuação destas três áreas. Os próprios mercados criam problemas na sociedade para os quais não apresentam soluções, de tal modo que os governos devem agir para solucioná-los (CLUNE, 1993). ${ }^{13}$

\subsection{O instrumentalismo jurídico}

${ }^{12}$ Seria possível explorar um paralelo entre as políticas preventivas de Clune e a sanção premial de Bobbio (2007).

${ }_{13}$ Considerando o Estado Social como o Estado das políticas públicas, poderia ser explorado paralelo entre a interdependência referida por Clune e os planos de ação governamental macro, meso e microinstitucional mencionados por Bucci (2013, cap. 3). Ainda que se olhe para a ação governamental (plano microinstitucional) ou os arranjos institucionais (plano mesoinstitucional), não se pode ignorar as determinações que impactam intensamente esses dois planos advindas do plano macroinstitucional, que engloba a política (politics) e o Estado. Nesse sentido, torna-se relevante situar as políticas públicas analisadas em sua realidade material e política específica, uma vez que o olhar para o plano macroinstitucional é indispensável para uma leitura política (politics) das políticas públicas (policies). 
Clune (1993, p. 08) afirma que o direito pode ser definido como um exercício de autoridade do Estado, sendo o poder estatal distintivo e soberano, por se constituir como a "autoridade com a atribuição de comandar as demais autoridades". Assim, os próprios incentivos criados pelo direito representam o uso da autoridade política inerente à criação normativa. Frequentemente o direito é utilizado como um instrumento político para auxiliar as políticas públicas nos problemas que essas almejam solucionar. Nas palavras de Clune (1993, p. 01): "governos aprovam leis e as utilizam para efetuar políticas públicas".

Isso tem paralelo com o que afirma Teubner (1988) sobre a instrumentalização do direito e particularmente o pós-instrumentalismo jurídico, a respeito do qual afirma que a reflexive law não se limita a descrever o objeto analisado, mas constitui e conforma o próprio objeto de estudo. Sobre a questão, Barcley e Birkland (1998) destacam a possibilidade do direito ser utilizado como uma ferramenta das políticas públicas. Lasswel e McDougal (1992), de maneira similar, afirmando que as necessidades contemporâneas requerem um "uso consciente e deliberado do direito como instrumento de políticas públicas". Apesar da sua crescente ascensão, a instrumentalização do direito recebeu diversas críticas.

Embora o direito tenha relação com restringir a liberdade de alguns para expandir a liberdade de outros - como o direito à propriedade, a redistribuição de renda, direito do empregador e dos empregados - a vinculação com a política pública talvez seja o ponto em que o direito busque um maior equilíbrio entre liberdade e restrição (CLUNE, 1993). Instituições jurídicas são construídas com o objetivo, entre outros, de atender as demandas impostas pelas políticas públicas, de modo que os resultados sociais e econômicos são alcançados mediante instrumentos jurídicos. Isso porque, toda política pública deve ser implementada a partir de um conjunto de técnicas legais e uma série de tomadas de decisões jurídicas (CLUNE, 1993).

Neste ponto, podemos visualizar a intensa interação entre instituições jurídicas, política (politic) e políticas públicas (policy), considerando as afirmações de Lowi de que não é apenas a politics que forma a policy mas, frequentemente, a policy acaba formando e determinando a politics. Dito de outro modo, embora se costume afirmar que o âmbito político determinará as políticas públicas, muitas vezes observamos que as políticas públicas ocupam um espaço tão grande no governo que elas acabam determinando os rumos e resultados da política (LOWI, 1972). Em texto específico em que aborda o tema DPP, Lowi destaca que os termos law e public policy possuem datas e origens diferentes de surgimento. Segundo o autor, enquanto law foi a palavra da fundação dos EUA (governo das leis e não dos homens), a palavra public policy é mais recente, surgindo apenas no século XIX. A expressão public policy, que inicialmente era um sinônimo de opinião pública, foi transformando-se, no final do século XIX, e ganhou o significado de "o que o governo faz" (LOWI, 2003). 


\subsection{Permanência das instituições no tempo}

Uma característica importante na relação entre direito e políticas públicas refere-se à permanência das estruturas legais no tempo (CLUNE, 1993). As políticas públicas dificilmente são criadas do zero, tendo em vista que partem de uma estrutura institucional anteriormente posta, que ampliará ou restringirá suas chances de efetividade. Em outras palavras, o êxito de uma política pública é mediado pelas instituições jurídicas consolidadas na estrutura estatal. ${ }^{14}$

Entretanto, é necessário que as estruturas legais, que permanecem no tempo, possuam alguma capacidade de mudança e adaptação, em vista das novas necessidades criadas pelas alterações no campo econômico. $\mathrm{O}$ caráter de permanência das instituições não é sinônimo de imutabilidade, considerando as transformações recorrentes na área econômica e social que, por vezes, resultam em reformas institucionais no aparato estatal (CLUNE, 1993)..$^{15}$

Mais um ponto de conexão entre o direito e a política é aquele que se nota quando observamos que uma mudança no ambiente político resulta em relevantes alterações no plano normativo. Assim, a produção normativa auxilia na legitimação e suporte dos novos rumos ditados por um governo, ao mesmo tempo em que o exercício legiferante precedente cria restrições para a consolidação dessas mudanças.

Por fim, considerando que as instituições envolvidas com a implementação e formulação das políticas públicas não são similares e possuem divergências internas marcantes, Clune (1983) propõe uma diferenciação entre as instituições de múltiplos propósitos e as instituições de propósito único. Essas seriam mais eficazes na implementação de políticas públicas do que as instituições de múltiplos propósitos, dado que a multiplicidade de objetivos e responsabilidades das últimas tornariam os seus recursos de implementação mais diluídos.

\footnotetext{
${ }^{14}$ Poderia ser examinado eventual paralelo entre essa noção e a afirmação de Bucci (2013) a respeito do caráter de impessoalidade que as instituições ganham com o passar do tempo, auxiliando essa permanência institucional.

${ }^{15}$ Apesar de Clune não utilizar o conceito de path dependency, seria interessante investigar possíveis relações entre a permanência das instituições e essa noção, segundo a qual as decisões tomadas no momento em que as instituições são formadas influenciam de maneira relevante no futuro funcionamento dessas, considerando o provável constrangimento criado por elas, que dificultaria eventuais mudanças em sua estrutura (GAINS; JOHN; STOKER, 2005). Nas palavras de Pierson (2004): "os custos de mudar de uma alternativa para outra irão, em certos contextos sociais, aumentar consideravelmente ao longo do tempo". Assim, a dependência da trajetória seria relevante para delimitar a direção e o desenvolvimento posterior das instituições e, consequentemente, das políticas públicas.
} 


\section{QUADROS DE ANÁliSE E TÉCNICAS DE PESQUISA PARA A ABORDAGEM DIREITO} E POLÍTICAS PÚBLICAS

Clune (1983) ressalta a existência de diferentes quadros de análise úteis para a abordagem direito e políticas públicas. $\mathrm{O}$ autor afirma que uma teoria ou quadro de análise presta-se à simplificação, organização e sistematização de uma realidade profundamente complexa. Dessa forma, um quadro representa a possibilidade de jogar luz a sobre um ponto específico do objeto de pesquisa. Dado o limite de espaço do presente artigo, discorreremos apenas sobre alguns dos frameworks abordados pelo autor.

\subsection{Análise institucional comparativa}

Um dos quadros de análise utilizados pelo próprio Clune em alguns de seus artigos é o chamado Comparative Institucional Analysis, que pode ser definido, essencialmente, como uma "rigorosa investigação sobre as vantagens relativas de várias instituições jurídicas e sociais em alcançar determinados objetivos em políticas públicas, especialmente bem estar em escala ampla." (CLUNE; 1993; p.27). Desse modo, considera-se tanto o aspecto político quanto o técnico, a partir da pesquisa sobre a capacidade política e regulatória das instituições, bem como os processos de tomada de decisão vinculados a essas. Dito de outra forma, o quadro analisa a capacidade das instituições de criar políticas públicas efetivas e a capacidade de influenciar o comportamento dos indivíduos por meio de incentivos (1993). O foco dessa análise consiste, de maneira suscinta, em decidir sobre qual instituição será mais efetiva para tomar a decisão e implementar a política pública, ou seja, "to decide who decides". Esse quadro de análise foi inicialmente desenvolvido e difundido por outro professor da Wisconsin Law School, Neil Komesar (1994). Aplicações e abordagens desse quadro podem ser encontrados em Clune (1984) e Komesar (1994).

\subsection{Análise empírica de políticas públicas}

Outra tradição analítica muito utilizada na área de direito e políticas públicas é a Empirical/Analytical Policy Analysis, que estuda, a partir de pesquisas empíricas, os problemas que as políticas públicas pretendem solucionar, pela perspectiva dos objetivos previamente determinados pela ação governamental e o seu consequente impacto jurídico. Todavia, o autor aponta a fragilidade de empreender uma simples análise do impacto legal das políticas públicas, considerando os múltiplos fatores que influenciam a vida social, bem como as diversas mediações existentes nessa relação, tais como os constrangimentos de ordem cultural e organizacional, as especificidades de cada política pública setorial e os diferentes contextos existentes. Em outras palavras, é possível que o impacto produzido por uma política pública 
seja resultado de outras determinações não relacionadas diretamente com a sua base normativa (CLUNE, 1993).

Analisar a efetividade de uma política de segurança pública, por exemplo, a partir de critérios como o número de prisões realizadas pode ser extremamente falho, na medida em que esse critério não representa uma real efetividade da política referida. Se pensarmos no Brasil, podemos compreender melhor a questão, considerando que possuímos a terceira maior população carcerária do mundo e, ao mesmo tempo, temos mais de 60 mil homicídios registrados anualmente (BRASIL, 2019). Outro exemplo é o Programa Bolsa Família, pois a efetividade dessa política frequentemente está vinculada à criação de um cadastro de beneficiários, instituído por norma infra-legal, sendo que a existência deste cadastro costuma ser explicitado em trabalhos que não sejam jurídicos (BUCCI, 2019). Desse modo, a utilização de alguns critérios para analisar o impacto jurídico de uma política pode se mostrar falho se não utilizado de forma cautelosa.

\subsection{Teoria do empoderamento e transferência de desenhos institucionais úteis entre áreas de políticas públicas}

Clune coloca foco em um framework que, segundo o próprio autor, possibilita acoplar políticas públicas, pesquisa, prática e ética. Clune refere-se à The Theory of Empowerment and the Tranfer of Useful Institucional Design from one Area of Policy to Another (Teoria do Empoderamento e da Transferência de Desenhos Institucionais Úteis entre Áreas de Políticas Públicas). O quadro de análise em questão tem como premissa a importância do empoderamento dos cidadãos por meio políticas públicas - principalmente os indivíduos que possuem pouco capital cultural e econômico. Empoderamento, nessa concepção teórica de políticas públicas, é entendido como a distribuição de cinco tipos de recursos: direitos, informação, renda, acesso às organizações sociais relevantes e poder político (CLUNE, 1993). Essa distribuição, por intermédio das políticas públicas, permitiria uma posição mais ativa dos indivíduos.

Esse quadro de análise resgata a ideia já debatida sobre a característica de centralização e descentralização das políticas públicas, em vista da necessidade de estimularem as práticas de condutas socialmente desejáveis. Assim, para serem efetivas: "instituições jurídicas combinam uma forte direção centralizada com um alto grau de delegação, descentralização e mesmo empoderamento" (CLUNE, 1993, p. 34). Partindo dessa perspectiva, é possível pensar em arranjos institucionais que estabeleçam uma direção centralizada por parte do Estado e, ao mesmo tempo, uma delegação de certas atividades aos indivíduos da sociedade.

Arranjos institucionais desse tipo guardam sintonia com a ideia, defendida por Clune, de uma implementação que não seja top/down, mas em outro sentido, garanta participação e empoderamento dos indivíduos. Isso não significa, entretanto, que esses novos modelos de implementação não precisariam de uma 
burocracia capacitada e de instituições funcionais à política. Aplicações desse quadro de análise podem ser encontrados em Clune e White (1988) e Handler (1989).

\section{AS DIFERENTES ESPÉCIES NORMATIVAS E SUAS RELAÇÕES COM AS POLÍTICAS PÚBLICAS: APROXIMAÇÕES ENTRE KREIS E CHRISTENSEN E KIYOUNG KIM}

Kreis $^{16}$ e Christensen ${ }^{17}$ elaboram um conceito inclusivo de direito e políticas públicas, afirmando que se foca em problemas (policy problems) e no intercâmbio de “(I) opções políticas discricionárias, permeadas por restrições sociais e políticas, que buscam lidar com os problemas por meio de instrumentos legais (constitucional, estatutário, regulatório e judicial); (II) prática administrativa (procedimento e rotina); (III) impacto econômico, social, político e jurídico" (KREIS; CHRISTENSEN, 2013 $)^{18}$. Os autores destacam as diversas espécies normativas que influem sobre as políticas públicas, diferenciando-as em normas constitucionais, estatutárias, regulatórias e judiciais. Cada norma guardaria sua especificidade enquanto instrumento jurídico utilizado pelas políticas públicas para a resolução de problemas e em relação à diversidade de opções disponíveis para a construção das soluções almejadas. Sublinhe-se que a dimensão jurídica das políticas públicas - que garante sua existência formal - é apenas uma das fases necessárias para que essas alcancem os objetivos previamente estabelecidos.

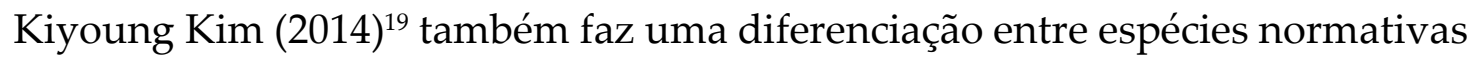
relacionadas com as políticas públicas, apontando a existência - teorizada no direito administrativo - de quatro tipos de normas que direcionam o comportamento dos administradores e policymakers, quais sejam, normas regulatórias, constitucionais, estatutárias e common law. As primeiras estariam relacionadas com as regras emitidas pelas agências regulatórias, sendo classificadas como normas secundárias, por serem criadas pelo Poder Executivo, a partir de uma delegação legislativa. Essas poderiam ser contrapostas às normas estatutárias, pois as últimas são criadas pelo Poder Legislativo e possuem a função de articular problemas e soluções (KIM, 2014). O fato de a norma estatutária e a norma regulatória emanarem de poderes distintos diz muito sobre a dinâmica interna de produção de cada uma, considerando o funcionamento peculiar do Executivo e do

16 Professor visitante de Direito da Chicago-Kent College of Law. Concluiu seu doutorado em Ciência Política e Administração Pública na University of Georgia, nos EUA.

17 Professor assistente de Administração Pública no Departamento de Administração Pública e Políticas Públicas, Escola de Assuntos Públicos e Internacionais na University of Georgia, nos EUA.

${ }_{18} \mathrm{O}$ artigo de Kreis e Christensen foi indicado, inicialmente, por Fernando Alves Gomes, a quem agradecemos.

${ }^{19}$ Professor de Direito da Chosun University, na Coreia do Sul. 
Legislativo no tocante à produção normativa ${ }^{20}$. Assim, as especificidades de cada uma delas enquanto guias do comportamento dos administradores e policymakers devem ser consideradas.

Assim, a depender da arena de onde a política pública emane - Legislativo, Executivo ou Judiciário - haverá relevantes diferenças na efetividade da ação governamental, pois os arranjos institucionais dessas arenas servem de mediadores, que restringem ou ampliam a eficácia das políticas públicas. A visualização dos pontos de veto e das distintas arenas que se relacionam com as políticas públicas auxiliam na noção de instrumentalização do direito, pois se podemos utilizar as técnicas jurídicas com o objetivo de solucionar determinados problemas, a compreensão dos eventuais obstáculos presentes em cada arena pode ajudar na elaboração de soluções e estratégias mais precisas.

Nesse tema, há referência, ainda, às decisões do Poder Judiciário e ao controle judicial das políticas públicas. Tanto Kiyoung Kim e Kreis e Christensen as mencionam, com a diferença de que, enquanto Kim refere-se a common law, Kreis e Christensen usam o termo judicial law para denominarem o mesmo tipo de decisão.

Por fim, a Constituição, enquanto norma hierarquicamente superior, que define os fundamentos do Estado e dispõe sobre sua organização, estabelece limites jurídicos que, idealmente, deverão ser observados por todas as autoridades envolvidas com a formulação e implementação das políticas públicas (KIM, 2014). As normas constitucionais possuem relação direta com o que Lowi denominou de políticas constitutivas (1972), ou seja, as regras que definem como as regras serão elaboradas, além de moldarem as arenas em que ocorrerão as disputas políticas.

A fiigura abaixo, elaborada por Kreis e Christensen (2013), ilustra as etapas da política pública e as expressões jurídicas pertinentes.

20 Essa questão tem contato com pesquisas que exploram diferenças encontradas na fase de produção da norma, sob a perspectiva da abertura para o lobby, que seria maior no Legislativo se comparado ao Poder Executivo (CESÁRIO, 2016), ou da existência de pontos de vetos no Poder Legislativo, como o sistema bicameral ou as votações em comissões temáticas. 
Figura 1 - Etapas da política pública e as expressões jurídicas pertinentes

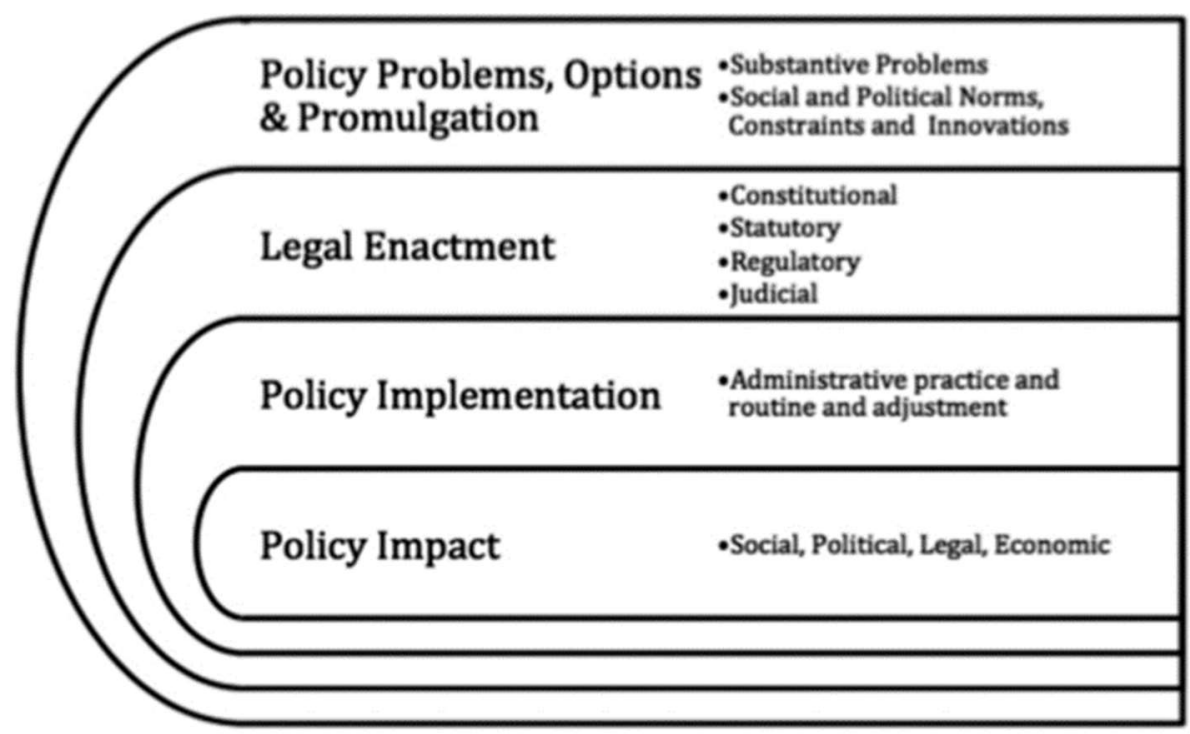

Fonte: Kreis e Christensen (2013)

As diferentes espécies normativas - constitucional, estatutária, regulatória e judicial - não estão isoladas no processo de formulação e implementação das políticas públicas, mas sim interligadas e exercendo efeitos umas sobre as outras. Assim como o ciclo de políticas é um instrumento didático, pois na prática o processo nem sempre ocorre de maneira uniforme e cronológica, as diferentes normas que influenciam na ação governamental também podem aparecer em diferentes fases desse ciclo. Isso porque, o processo de formulação e implementação de uma política é permeado por uma grande complexidade - envolvendo diferentes atores estatais e não estatais, diversas instituições e restrições jurídicas e econômicas - e está em constante movimento, não sendo possível visualizá-lo de forma estática. Esse movimento está vinculado à relação existente entre direito e política, que demanda uma análise dinâmica da produção normativa.

\section{DIREITO E POLÍtICAS PÚblicAs: FRONTEIRAS DISCIPLINARES E METODOLÓGICAS}

1. Seriam os tribunais formuladores de políticas públicas? Um debate entre duas áreas do conhecimento

Mesmo que pesquisadores de políticas públicas e da área de direito público compartilhem algumas disciplinas e investiguem questões similares, há 
divergências marcantes entre eles. A principal delas, apontada por Barcley ${ }^{21} \mathrm{e}$ Birkland ${ }^{22}$, relaciona-se à função dos tribunais enquanto policymakers, assim resumidas: “a) a função das cortes na elaboração de políticas públicas; b) a compreensão do significado do processo; c) hipóteses sobre os atributos típicos das instituições vinculadas às políticas públicas" (BARCLAY; BIRKLAND, 1998).

Os pesquisadores da área jurídica sofreram grande influência de Robert Dahl, que definiu a Suprema Corte dos EUA como uma policymaking institution; "os tribunais agem para criar, articular e legitimar as políticas públicas do governo, de tal modo que cumprem o papel de uma policymaking institution" (BARCLAY; BIRKLAND, 1998, p. 228). A partir dessa afirmação, os juristas defendem que os tribunais criam as políticas públicas através de casos jurídicos e da interpretação das leis existentes, de tal forma que poderiam ser classificados como formuladores de políticas públicas. A função dos tribunais teria grande impacto principalmente em duas fases do processo de policymaking: implementação e formação da agenda.

A Suprema Corte dos Estados Unidos representaria um exemplo típico do desempenho dessa função, considerando suas decisões sobre liberdades civis, união homoafetiva, políticas educacionais, entre outros temas. Entretanto, isso não impede os pesquisadores da área jurídica de reconhecerem a eventual incapacidade dos tribunais para implementarem suas decisões, retomando a clássica afirmação de Hamilton de que o Judiciário é o Poder "sem bolsa nem espada" (BARCLAY; BIRKLAND, 1998).

Alguns autores da área de políticas públicas, na mesma linha, concordam que em certos casos os tribunais podem cumprir o papel de formuladores de políticas (BARCLAY; BIRKLAND, 1998). ${ }^{23}$

Mas em sentido diverso, há estudiosos de políticas públicas e autores de direito público que não defendem esse entendimento, sustentando que os tribunais não são policymakers, mas apenas reagem às políticas públicas elaboradas por outras instituições (BARCLAY; BIRKLAND, 1998).

\footnotetext{
${ }^{21}$ Scoot Barclay é Professor no Departamento de Ciência Política da State University of New York.

22 Thomas Birkland é Professor do Departamento de Ciência Política e do Departamento de Administração Pública da State University of New York.

${ }^{23}$ E há, ainda, posições peculiares de outros autores de referência no campo. Sabatier critica o modelo de múltiplos fluxos de Kingdom, alegando que esse framework dá pouca importância às burocracias e tribunais. Entretanto, essa crítica é relativizada pelo fato de que o modelo de Sabatier, o Advocacy Coalition Framework, também negligencia a função dos tribunais, por que apesar de reconhecê-los como importantes atores políticos, considera-os agentes passivos no processo de elaboração de políticas (BARCLAY; BIRKLAND, 1998).
} 


\section{O estudo das políticas públicas no direito e as diferentes técnicas de pesquisa}

O crescimento das pesquisas sobre políticas públicas e o estudo dessas na seara jurídica alterou as tradicionais barreiras disciplinares, criando um solo fértil para o surgimento de diálogos interdisciplinares. Essa afirmação pode ser evidenciada pelo fato de que muitos juristas começam a utilizar métodos das ciências sociais para o desenvolvimento de suas análises jurídicas (KREIS; CHRISTENSEN, 2013). Entretanto, essa abertura interdisciplinar é um fenômeno recente, considerando que os currículos das faculdades de direito costumam conter disciplinas tradicionais, dogmáticas e autocentradas. Isso porque, ainda há alguma preocupação com uma pretensa pureza do objeto de estudo do direito, o que torna a pesquisa sobre políticas públicas não apenas negligenciada, mas por vezes, evitada (BAXTER, 1985).

A abertura para o diálogo interdisciplinar ${ }^{24}$ na abordagem DPP torna possível a construção de análises empíricas, normativas e teóricas (KREIS; CHRISTENSEN, 2013). Sobre as análises empíricas de políticas públicas na área da sociologia do direito, Deborah Hensler (2003) entende que o que as diferencia das demais análises é que essas buscam construir suas conclusões a partir dos fatos, e não de análises normativas ou de deduções formais. Podemos apontar, também, pesquisas de caráter histórico em direito e políticas públicas, como a de Molly Selvin (2013).

Lasswell e McDougal (1943) abordam os desafios para o ensino do direito sintonizado com as políticas públicas e o interesse público. Afirmam que os esforços para integrar o direito às outras ciências sociais costumam ter pouco êxito, em virtude da "falta de claridade sobre o que está sendo integrado, e como, e para quais propósitos" (1943). É interessante observar como a crítica realizada pelos autores há mais de 70 anos ainda é, em alguns casos, bem atual.

Ao analisarem os princípios da organização curricular do direito direcionados às políticas públicas, Lasswell e McDougal (1943) afirmam a necessidade de "todas as estruturas jurídicas, doutrinas, e definições serem ensinadas, avaliadas e recriadas de acordo com os valores democráticos básicos", pois isso permitiria dar um significado operacional aos conceitos transmitidos, na medida em que os vincularia aos contextos institucionais macro nos quais estão inseridos. Desse modo, uma reorganização curricular dos cursos de Direito, levando em conta a relevância das políticas públicas, deveria ter como norte três princípios: princípio de valor; princípio de habilidade e princípio de influência.

${ }^{24}$ Sobre o tema, ver os artigos de Rizzi e Bambini, além de Brunet, neste dossiê. 


\section{Crítica à separação entre política e políticas públicas}

Ainda sobre as pesquisas empíricas no âmbito da sociologia do direito, Sarat e Silbey (1988) criticam os que defendem a neutralidade dos estudos de políticas públicas, como uma mera técnica que permite ligar meios aos fins, desconsiderando o aspecto político inerente a esses estudos. Assim, tecem uma crítica àqueles que diferenciam policy de politcs no sentido de apontar a análise de políticas públicas como um campo apolítico. A preocupação com um governo eficiente, desse modo, seria uma questão de técnicas eficientes de administração, desvinculando-a da política com P maiúsculo (SARAT; SILBEY, 1988). Em sentido similar, Deborah Stone afirma que a "análise de políticas públicas é um argumento político" (SARAT; SILBEY, 1988, p.101), de tal modo que discussões sobre políticas públicas podem, com frequência, obscurecer os conflitos políticos subjacentes às ações governamentais, mas não eliminá-los.

"Politizar" a análise de políticas públicas implica reconhecer que as normas jurídicas que dão suporte às policies são formas de cristalização da disputa entre interesses divergentes. Investigar a imbricação entre direito, políticas públicas e política pode contribuir para evidenciar os conflitos políticos, as posições de cada um dos vários interesses implicados e as formulações jurídicas pertinentes à política pública em questão.

\section{CONSIDERAÇÕES FINAIS}

A abordagem DPP possui um instrumental analítico que permite a visualização de problemas não evidenciados pela análise jurídica tradicional. Isso não significa substitui-la, uma vez que ela permanece indispensável, mas ampliar o leque de considerações para a compreensão de problemas públicos complexos e de escala ampla, em que se revela particularmente útil o descortínio da imbricação do elemento jurídico com questões políticas. Pode-se explorar o diálogo do Direito com a Ciência Política e a Análise de Políticas Públicas para visualizar as normas não enquanto objetos prontos e acabados, mas, dando um passo atrás, observar o seu processo de formação e enxergá-las pelo ângulo das disputas políticas travadas em torno de interesses divergentes. Ganham grande relevo, assim, as instituições e o desenho jurídico-institucional (BUCCI; COUTINHO, 2017; STUCHI, 2018).

Nesse sentido, o resultado da pesquisa foi útil para evidenciar, na bibliografia selecionada, a valorização do diálogo entre autores da Direito, Policy Analysis e Ciência Política; a interlocução entre áreas permitindo uma lapidação das lentes utilizadas. Entretanto, para dar voz à crítica de Lasswell (1943), é necessário ter em mente o que deve ser objeto desse diálogo, como construi-lo e, principalmente, quais os propósitos que levam a estabelecer essa interlocução. Como vimos, os trabalhos relatados neste artigo apontam algumas possibilidades, especialmente nos seguintes temas: a) instrumentalismo jurídico; b) quadros de análise úteis à 
abordagem DPP; c) a relação entre diferentes espécies normativas na formulação e implementação das políticas públicas; d) permanência das instituições jurídicas no tempo; e) os tribunais enquanto policymakers; f) teorias subjacentes ao pensamento jurídico e à policy analysis; g) crítica à separação entre política (politics) e políticas públicas (policy); h) o ensino de políticas públicas nos cursos de direito; i) políticas públicas no contexto do Estado Social. A quantidade de temas abordados, ainda que de forma sintética, indica uma agenda de pesquisa fértil a ser desenvolvida, que pode representar caminhos para a abordagem DPP.

Destacando a contribuição de William Clune, um dos pontos a sublinhar é a demarcação da política pública no contexto do Estado Social, o que implica considerar o Estado, não enquanto aparato puramente abstrato, mas enquanto Estado determinado histórica e politicamente, vinculado a uma fase específica da organização econômica capitalista. Neste ponto, encontramos a política pública e o direito não como concepções abstratas, que pairam no ar, mas com os pés no chão, ou seja, com uma materialidade política e histórica cuja compreensão requer entendimento sobre as conexões entre a policy e as politics. É nesse plano que se poderão responder às críticas sobre a despolitização das políticas públicas. E, a despeito do cuidado que se deve ter na transposição de caminhos institucionais pensados nos EUA, considerando a tradição jurídica anglo-americana e suas especificidades, essa linha de investigação é ainda mais pertinente num país em desenvolvimento, como o Brasil.

Mas, mais uma vez com Clune, é necessário precaver-se contra a politização traduzida em consequencialismo raso, embutido no instrumentalismo jurídico próprio das políticas públicas. O sentido da abordagem DPP é inspirar a produção de políticas públicas que realizem direitos fundamentais não apenas como entrega de serviços públicos, mas como construções institucionais que reflitam a organização democrática do poder.

À guisa de fecho, registre-se que um dos maiores desafios para a realização dessa pesquisa foi encontrar artigos que tratassem, especificamente, do direito e das políticas públicas em termos metodológicos e disciplinares, para além da análise de políticas setoriais. Essa dificuldade evidencia que a despeito de haver, no âmbito internacional, produção de cerca de trinta anos, trata-se de uma abordagem ainda em construção. Assim como ocorre no Brasil.

\section{REFERÊNCIAS}

ABRAMOVICH, V. El rol de la justicia en la articulación de políticas y derechos sociales, La revisión judicial de las políticas sociales. In: ABRAMOVICH, V; PAUTASSI, L. (eds.). Estudio de casos. Buenos Aires: Editores del Puerto, 2009. 
ABRAMOVICH, V. Una aproximación al enfoque de derechos en las estrategias y políticas de desarrollo. Revista de la CEPAL, n. 88 (LC/G.2289-P/E), 2006.

BARCLAY, S.; BIRKLAND, T. Law, Policymaking, and the Policy Process: Closing the Gaps. Policy Studies Journal, n. 26, 1998, p. 227-243.

BAXTER, L. G. Legal Education and Public Policy. Natal University Law and Society Review, n. 1, 1985, p. 15-31.

BOBBIO, Norberto. Da estrutura à função: novos estudos de teoria do direito. Tradução: Daniela Beccaccia Versiani. São Paulo: Manole, 2007.

BUCCI, Maria Paula Dallari. Fundamentos para uma Teoria Jurídica das Políticas Públicas. São Paulo: Saraiva, 2013.

BUCCI, Maria Paula Dallari. O conceito de política pública em direito. In: BUCCI, Maria Paula Dallari (org.). Políticas Públicas: Reflexões sobre o Conceito Jurídico. São Paulo: Saraiva, 2006.

BUCCI, Maria Paula Dallari. Método e aplicações da abordagem Direito e Políticas Públicas. REI-Revista Estudos Institucionais, v. 5, n. 3, 2019.

BUCCI, Maria Paula Dallari; COUTINHO, Diogo. Arranjos jurídico-institucionais da política de inovação tecnológica: uma análise baseada na abordagem de direito e políticas públicas. In: COUTINHO; FOSS; MOUALEM (orgs.). Inovação no Brasil: avanços e desafios jurídicos e institucionais. São Paulo: Blucher, 2017.

BRASIL. IPEA. Atlas da violência 2019. Organizadores: Instituto de Pesquisa Econômica Aplicada; Fórum Brasileiro de Segurança Pública. Brasília: Rio de Janeiro: São Paulo: 2019.

BRUNET, Emiliano. Sobre a abordagem Direito e Políticas Públicas (DPP) em um curso de graduação em Direito: contribuição crítica para a construção de um programa. REI-Revista Estudos Institucionais, v. 5, n. 3, 2019.

CHRISTENSEN, R. K. International Nongovernmental Organizations: Globalization, Policy Learning, and the Nation-State. International Journal of Public Administration, v. 29, 2007, p. 281-303. 
CLUNE, William H. Law and Public Policy: map of an area. Review of Public Affairs, University of Wisconsin-Madison, 1993. Working Paper No. 6.

CLUNE, William. H. A Political Model of Implementation and Implications of the Model for Public Policy, Research, and the Changing Roles of Law and Lawyers. Iowa L. Rev, v. 69, n. 47, 1983.

CLUNE, William. H. Legal Disintegration and a Theory of the State. German L.J., v. 12, n. 186, 2011.

CLUNE, William; WHITE, Paula. School-Based Management. Institutional Variation, Implementation, and Issues for Further Research. New Brunswick, NJ: Rutgers University, Consortium for Policy Research in Education, 1988.

COUTINHO, Diogo R. O direito nas políticas públicas. In: MARQUES, Eduardo; FARIS, Carlos Aurélio Pimenta de (orgs.). A política pública como campo multidisciplinar. São Paulo: Ed. Unesp/Ed. Fiocruz, 2013.

HANDLER, Joel F. Dependent People, the State, and the Modern/Postmodern Search for the Dialogic Community. UCLA L. Rev., v. 35, n. 999, 1989.

HARRINGTON, C. B; CARTER, L. H. Administrative law and politics: Cases and comments. 4. ed. Washington, DC: CQ Press, 2009.

HENSLER, Deborah R. Reflections on the Use of Empirical Research in Legal Policy Reform. Newcastle L. Rev., v. 7, n. 1, 2003.

KIM, Kiyoung. The Relationship between the Law and Public Policy: Is it a ChiSquare or Normative Shape for the Policy Makers. Social Sciences, v. 3, n. 4, 2014, p. 137-143.

KERWIN, C. M.; FURLONG, S.R. Rulemaking: How government agencies write law and make policy. 4. ed. Washington, DC: CQ Press, 2011.

KINGDON, John. Agendas, alternatives, and public policies. 3 ed. Nova York, Harper Collins, 2003.

KOMESAR, Neil K. Imperfect alternatives: choosing institutions in law, economics and public policy. Chicago University Press: Chicago, 1994. 
KREIS, A. M.; CHRISTENSEN, R. K. Law and Public Policy. Policy Stud J, n. 41, 2013, p. 38- 52.

LASSWELL, Harold D; MCDOUGAL, Myres S. Legal education and public policy: Professional training in the public interest. Yale Lj, v. 52, n. 203, 1943.

LASSWELL, Harold D; MCDOUGAL, Myres S. Jurisprudence for a free society: studies in law, science, and policy. v. 1. Martinus Nijhoff Publishers, 1992.

LOWI, Theodore J. Law vs. Public Policy: A Critical Exploration. Cornell Journal of Law and Public Policy, v. 12, n. 3, 2003.

MANTILLA, Gorki Gonzales. La Ensenanza del Derecho como Politica Publica. Derecho PUCP, v.65, n. 285, 2010.

MCDOUGALL, Harold A. Lawyering and Public Policy. Journal of Legal Education, vol. 38, 1988, p. 369.

PAUTASSI, L. C. La articulación entre políticas públicas y derechos, vínculos difusos. In: ERAZO, X; MARTIN, P.; OYARCE, H. (Eds.) Políticas públicas para un Estado social de derechos. El Paradigma de los Derechos Universales. Santiago de Chile: lom, 2009 (2009a).

PAUTASSI, L. El aporte del enfoque de derechos a las políticas sociales. Una breve revisión", documento presentado en el taller de expertos Protección social, pobreza y enfoque de derechos: vínculos y tensiones. Comisión Economica para América Latina y el Caribe (CEPAL). Santiago de Chile, 2009 (2009b).

PIERSON, Paul. Placing politics in time. History, Institutions and Social Analysis. Princeton University Press, 2004.

SARAT, A.; SILBEY, S. The pull of the policy audience. Law \& Policy, n. 10, 1988, p. $97-166$.

SELVIN, Molly. The History of Contemporary Law and Policy. Am. J. Legal Hist., n. 53,2013 , p. 502.

STUCHI, Carolina Gabas. Uma contribuição do direito para a análise de políticas públicas: o caso do sistema único de assistência social (SUAS). XI Encontro da АBCP, 2018. 
O ESTADO DA ARTE DA ABORDAGEM DIREITO E POLÍTICAS PÚBLICAS EM ÂMBITO INTERNACIONAL: PRIMEIRAS APROXIMAÇÕES

TEUBNER, Gunther. Dilemmas of law in the welfare state. De Gruyter/European University Institute, 1988.

YOSIFON, David. The Public Choice Problem in Corporate Law: Corporate Social Responsability After Citizens United. North Carolina Law Review, n. 89, 2011, p. 198-246. 